\title{
Ezetimibe Markedly Reduces Hepatic Triglycerides and Cholesterol in Rats Fed on Fish Oil by Increasing the Expression of Cholesterol Efflux Transporters ${ }^{\text {[ }}$
}

\author{
Yuji Tanaka, Takanori Ikeda, Hiroshi Ogawa, and Toshinori Kamisako \\ Department of Clinical Laboratory Medicine, Kindai University Faculty of Medicine, Osakasayama, Osaka, Japan (Y.T., T.K.), and \\ Faculty of Human Sciences, Tezukayama Gakuin University, Sakai, Osaka, Japan (T.I., H.O.)
}

Received February 7, 2019; accepted April 30, 2020

\begin{abstract}
Besides diet therapy, hypolipidemic pharmacological therapy may be a crucial component of nonalcoholic fatty liver disease (NAFLD) treatment. Ezetimibe may be a promising drug for treatment of NAFLD. $n-3$ polyunsaturated fatty acids, which are abundant in fish oil, reduce serum and hepatic cholesterol and triglycerides in rodents. The aim of this study was to examine the combined effects of dietary fish oil and ezetimibe on lipid metabolism in rats. Seven-week-old male Sprague-Dawley rats were allocated to four different diets containing 1) $10 \%$ soybean oil (C), 2) $10 \%$ fish oil (F), 3) $10 \%$ soybean oil $+0.005 \%$ ezetimibe, and 4$) 10 \%$ fish oil $+0.005 \%$ ezetimibe $(F+E)$ for 4 weeks, when the liver, jejunum, blood, and fecal samples were collected. Compared with the $\mathrm{C}$ group, the $\mathrm{F}+\mathrm{E}$ diet decreased hepatic triglycerides and cholesterol $84 \%$ and $86 \%$, but it did not increase fecal cholesterol. In liver, the expression of lipogenic enzymes was decreased in the $\mathrm{F}+\mathrm{E}$ diet, whereas $\beta$-oxidation-related genes were not increased. Abcg5/g8 mRNA expression was increased $1380 \% / 442 \%$ when ezetimibe was added to the $\mathrm{F}$ diet. These gene expression changes are related
\end{abstract}

to the decrease in hepatic lipids. In jejunum, Abcg5/g8 mRNA was increased $244 \% / 841 \%$ when ezetimibe was added to the $\mathrm{F}$ diet. Hepatic induction of Abcg5/8 rather than intestinal induction correlates with the marked decrease in liver cholesterol when ezetimibe was added to the $F$ diet. These data suggest that fish oil diet and ezetimibe in combination may be a beneficial therapy for NAFLD by increasing hepatic Abcg5/g8 and decreasing lipogenic genes.

\section{SIGNIFICANCE STATEMENT}

There is currently no single treatment for NAFLD. Thus, lifestyle modifications including dietary regulation and physical activity are also important options. In this study, ezetimibe, a cholesterol absorption inhibitor, was evaluated for the treatment of liver steatosis in rats fed on the different diets. We found that ezetimibe and fish oil in combination markedly improved fatty liver by increasing cholesterol efflux transporters. The combination therapy of fish oil agents and ezetimibe may be effective for NAFLD.

\section{Introduction}

Nonalcoholic fatty liver (NAFL) disease (NAFLD) is the hepatic manifestation of metabolic syndrome, and it includes a wide spectrum of liver diseases, ranging from NAFL to nonalcoholic steatohepatitis (NASH), which can progress to cirrhosis and hepatocellular carcinoma (Preiss and Sattar, 2008). The prevalence of NAFLD is rapidly increasing worldwide owing to the rising incidence of obesity. Apart from lifestyle modifications, including dietary regulation and exercise, several pharmacological agents have been evaluated for

This work was supported by JSPS KAKENHI [Grants 18K11006, 18K11115].

https://doi.org/10.1124/jpet.120.265660.

S This article has supplemental material available at jpet.aspetjournals.org. the treatment of NAFLD (Armstrong et al., 2016; Anushiravani et al., 2019; Aso et al., 2019). The American Association for the Study of Liver Diseases guidelines state that pioglitazone or vitamin $\mathrm{E}$ improves liver histology in patients with biopsy-proven NASH and therefore may be used to treat these patients (Chalasani et al., 2018). However, there are no established treatment options for NAFLD at present.

As patients with NAFLD frequently have dyslipidemia, lipid-lowering agents, including statins and ezetimibe, are expected to have potential therapeutic benefits in these patients. Ezetimibe is a cholesterol-lowering drug that inhibits NPC1L1, which is responsible for intestinal cholesterol absorption. Previous studies have demonstrated that ezetimibe prevents liver steatosis in mice fed on a high-fat diet and also in obese Zucker rats (Deushi et al., 2007; Ushio et al.,

ABBREVIATIONS: Abc, ATP-binding cassette transporter; ACC, acetyl-CoA carboxylase; Acox1, acyl-CoA oxidase 1; Asbt, apical sodiumdependent bile acid transporter; C, $10 \%$ soybean oil; Cl, confidence interval; E, $10 \%$ soybean oil + 0.005\% ezetimibe; EPA, eicosapentaenoic acid; F, $10 \%$ fish oil; FAS, fatty acid synthase; F+E, $10 \%$ fish oil + 0.005\% ezetimibe; HDL, high-density lipoprotein; HMG-CoA, 3-hydroxy-3methylglutaryl coenzyme A; Mdr2, multidrug resistance protein 2; NAFL, nonalcoholic fatty liver; NAFLD, NAFL disease; NASH, nonalcoholic steatohepatitis; NPC1L1, Niemann-Pick C1-Like 1; Ost, organic solute transporter; PCR, polymerase chain reaction; PPAR $\alpha$, peroxisome proliferator-activated receptor $\alpha$; SCD, stearoyl-CoA desaturase; SR-B1, scavenger-receptor class B, type 1; SREBP, sterol regulatory elementbinding protein. 
2013). The long-term administration of ezetimibe in human subjects improves the levels of serum lipids and alanine aminotransferase and also improves the histologic features of hepatic steatosis and inflammation, but not fibrosis (Park et al., 2011). In contrast, the Magnetic Resonance Imaging and Elastography in Ezetimibe Versus Placebo for the Assessment of Response to Treatment in NASH trial demonstrated that ezetimibe does not reduce liver fat content as quantified by the magnetic resonance imaging-derived proton density-fat fraction and does not improve liver histology in NASH (Loomba et al., 2015).

The n-3 polyunsaturated fatty acids, including eicosapentaenoic acid (EPA) and docosahexaenoic acid, are PPAR $\alpha$ ligands that are abundant in fish oil and have been shown to improve dyslipidemia and increase insulin sensitivity; therefore, they have been suggested as potential treatment options for NAFLD (Lombardo et al., 2007; Fakhrzadeh et al., 2010). A previous study demonstrated that an EPA-rich diet reduces the serum and hepatic triglyceride levels in leptin-deficient ob/ ob mice, which is a commonly studied model of obesity and liver steatosis (Sekiya et al., 2003). A study on rats demonstrated that the administration of a high-fat diet supplemented with fish oil lowers the hepatic triglyceride and cholesterol levels and improves hepatic steatosis, as observed in a group fed on a high-fat diet without fish oil supplementation (Yuan et al., 2016). A previous study on human subjects demonstrated that the administration of $2.7 \mathrm{~g}$ of EPA on a daily basis for 12 months reduces the serum levels of alanine aminotransferase and improves hepatic steatosis, as revealed by ultrasound imaging and analysis of the histologic features of hepatic steatosis, inflammation, and fibrosis (Tanaka et al., 2008). In contrast, a recent multicenter trial performed in North America demonstrated that ethyl-EPA, a synthetic polyunsaturated fatty acid, does not improve the levels of liver enzymes or the hepatic histology (Sanyal et al., 2014).

The present study aimed to examine the combined effects of fish oil and ezetimibe on the serum and hepatic levels of lipids by administering a menhaden fish oil diet or a control soybean oil diet, either unsupplemented or supplemented with ezetimibe, to male Sprague-Dawley rats for 4 weeks, after which the changes in the triglyceride and cholesterol status were analyzed by studying the lipid profiles and the expression of lipid and bile acid metabolism-related genes in the liver and jejunum.

\section{Materials and Methods}

Chemicals and Reagents. TRIzol reagent was purchased from Life Technologies (Tokyo, Japan), the PrimeScript RT reagent kit and TB Green Premix Ex Taq were purchased from Takara Bio (Shiga, Japan), and ezetimibe was purchased from Sigma-Aldrich (St. Louis, MO). Unless otherwise stated, all other chemicals were purchased from Wako Pure Chemical Industries (Osaka, Japan).

Diet. A control diet, containing $10 \%$ soybean oil as the fat source by weight, and three different experimental diets, containing $10 \%$ fish oil, $10 \%$ soybean oil with $0.005 \%$ ezetimibe, or $10 \%$ fish oil with $0.005 \%$ ezetimibe, were all purchased from Research Diets (New Brunswick, NJ). The composition of the control and experimental diets is shown in Supplemental Table 1.

Animals. Six-week-old male Sprague-Dawley rats were obtained from CLEA Japan (Shizuoka, Japan) and allowed to acclimate for 1 week in housing before beginning experiments. All rats were housed in the same animal care facility controlling for light, temperature, and humidity. Rats ( $n=7$ /group) were allocated to four different diets containing 1) $10 \%$ soybean oil (C; control group), 2) $10 \%$ menhaden oil (F; fish oil group), 3) $10 \%$ soybean oil plus $0.005 \%$ ezetimibe (E; ezetimibe group), and 4) $10 \%$ fish oil plus $0.005 \%$ ezetimibe $(\mathrm{F}+\mathrm{E}$; fish oil plus ezetimibe group) for 4 weeks. The dose of ezetimibe was chosen with reference to previous studies (Zheng et al., 2008). After 4 weeks, fecal samples were collected, blood was taken from the abdominal aorta under anesthesia with isoflurane, and livers were harvested as well as jejunum. Studies were approved by Kindai University Faculty of Medicine Animal Care and Use Committee.

Quantification of Serum, Hepatic, and Fecal Triglycerides and Cholesterol. Serum cholesterol and triglycerides were quantified by an AutoAnalyzer. Hepatic and fecal lipid content was extracted according to the method reported previously (Folch et al., 1957). The amount of triglycerides and cholesterol in the liver and feces was determined enzymatically using commercially available kits (Cholesterol E-test and Triglyceride E-test, respectively; Wako Pure Chemical Industries).

Histopathology. Liver tissue was fixed in $10 \%$ formalin and embedded in paraffin. H\&E was performed on each sample. Liver sections were stained with $\mathrm{H} \& \mathrm{E}$ and evaluated for hepatocellular steatosis.

RNA Isolation. Total RNA was isolated using TRIzol reagent according to the manufacturer's protocol (Life Technologies). The concentration of total RNA in each sample was determined spectrophotometrically at $260 \mathrm{~nm}$.

Real-Time Polymerase Chain Reaction. The mRNA expression level of 18s ribosomal RNA and cholesterol, bile acid, and fatty acid metabolism-related genes, including sterol regulatory elementbinding protein (SREBP)-1c, fatty acid synthase (FAS), acetyl-CoA carboxylase (ACC), stearoyl-CoA desaturase (SCD), fatty acid translocase (CD36), peroxisome proliferator-activated receptor $\alpha$ (PPAR $\alpha$ ), acyl-CoA oxidase 1 (Acox1), carnitine palmytoyltransferase 1, lowdensity lipoprotein receptor, scavenger-receptor class B, type 1 (SRB1), Niemann-Pick C1-like 1 (NPC1L1), ATP-binding cassette transporter g5 (Abcg5), Abcg8, Abca1, multidrug resistance protein 2 (Mdr2), cholesterol $7 \alpha$-hydroxylase (Cyp7a1), sterol-27 $\alpha$-hydroxylase (Cyp27a1), sterol-12 $\alpha$-hydroxylase (Cyp8b1), 3-hydroxy-3-methylglutaryl coenzyme A synthase (HMG-CoA synthase), HMG-CoA reductase, apical sodium-dependent bile acid transporter (Asbt), organic solute transporter $\alpha(\mathrm{Ost} \alpha)$, and Ost $\beta$, were quantified by SYBR realtime polymerase chain reaction (PCR). One of the primers was designed using Primer Express 2.0 (Applied Biosystems, Foster City, CA). Other primers were used as indicated in previous reports, and the sequences of these primers are shown in Supplemental Table 2 (Ballatori et al., 2005; Baumgardner et al., 2008; Chen et al., 2009; Boone et al., 2011; Gao et al., 2013; Wang et al., 2013; Sun et al., 2014; Tu et al., 2014; Madsen et al., 2015; Michihara et al., 2015; Kawashima et al., 2018; van Golen et al., 2018; Wei et al., 2018). The amplification reactions were performed in an ABI Prism 7900 sequence detection system (Applied Biosystems). The amount of mRNA was calculated using the comparative cycle threshold method, which determines the amount of target normalized to an endogenous reference. Each gene was normalized to 18 s ribosomal RNA.

Statistical Analysis. The number of animals was determined based on power analysis and previous reports (van Heek et al., 2001; Wang et al., 2014). The software package SYSTAT, version 11 (Systat Inc., Evanston, IL), was used for statistical analysis. All data were analyzed using one-way ANOVA, followed by Tukey's post hoc test. Differences were considered statistically significant at $P<0.05$.

\section{Results}

Weight Gain and Liver Weight. After feeding each diet for 4 weeks, weight gain did not differ among the groups. The liver weight was decreased $14 \%$ in the F-fed rats (95\% CI: 
TABLE 1

Body weight increase; liver weight; and serum, hepatic, and fecal concentrations of lipids.

Data are presented as means \pm S.D. (each group, $n=7$ animals). Data were analyzed by one-way ANOVA, followed by Tukey's post hoc test. Significance was set at $P<0.05$.

\begin{tabular}{lcccc}
\hline & $\mathrm{C}$ & $\mathrm{F}$ & $\mathrm{E}$ & $\mathrm{F}+\mathrm{E}$ \\
\hline Body weight increase (g) & $337.4 \pm 34.2$ & $316.6 \pm 19.0$ & $307.0 \pm 31.9$ & $306.1 \pm 40.5$ \\
Liver weight (g) & $22.6 \pm 2.5$ & $19.5 \pm 1.5^{a}$ & $19.8 \pm 2.0$ & $19.5 \pm 2.1^{b}$ \\
Serum triglyceride (mg/dl) & $392.4 \pm 157.1$ & $106.3 \pm 31.8^{c}$ & $241.0 \pm 102.0^{d}$ & $68.0 \pm 27.4^{e}$ \\
Serum total cholesterol (mg/dl) & $74.3 \pm 11.5$ & $50.1 \pm 8.7^{f}$ & $66.7 \pm 6.9$ & $47.4 \pm 9.5^{g}$ \\
Serum HDL cholesterol (mg/dl) & $25.9 \pm 3.1$ & $15.3 \pm 2.4^{h, i}$ & $27.8 \pm 1.2$ & $20.0 \pm 3.3^{j}$ \\
Liver triglyceride conc. (mg/g tissue) & $23.0 \pm 8.7$ & $13.9 \pm 5.4$ & $32.2 \pm 10.6$ & $3.6 \pm 1.2^{k}$ \\
Whole-liver triglyceride content (mg/whole liver) & $524.0 \pm 228.6$ & $270.5 \pm 115.4^{l}$ & $624.3 \pm 172.6$ & $70.8 \pm 28.4^{m}$ \\
Liver cholesterol conc. (mg/g tissue) & $1.6 \pm 0.5$ & $1.2 \pm 0.7$ & $1.9 \pm 0.9$ & $0.2 \pm 0.1^{n, o}$ \\
Whole-liver cholesterol content (mg/whole liver) & $36.0 \pm 10.2$ & $23.4 \pm 14.2$ & $36.3 \pm 12.5$ & $4.4 \pm 1.3^{p, q}$ \\
Fecal triglyceride (mg/g feces) & $0.8 \pm 0.2$ & $0.4 \pm 0.2^{r}$ & $0.6 \pm 0.2$ & $0.4 \pm 0.1^{s}$ \\
Fecal cholesterol (mg/g feces) & $2.5 \pm 0.5$ & $3.6 \pm 0.5^{t}$ & $3.2 \pm 0.7$ & $2.6 \pm 0.4^{u}$ \\
\hline
\end{tabular}

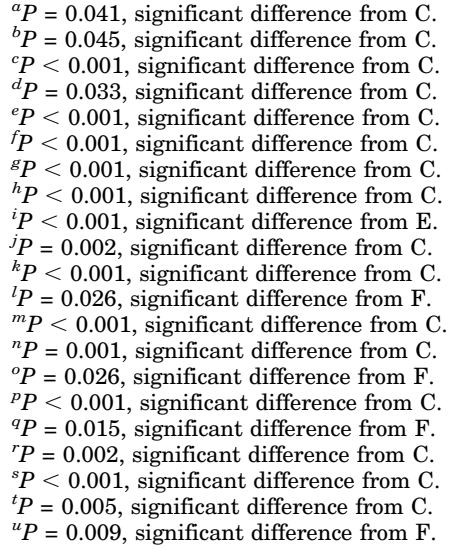

$9 \%-19 \% ; P=0.041)$ and $14 \%$ in the $\mathrm{F}+\mathrm{E}$ group $(95 \% \mathrm{CI}$ : $7 \%-21 \% ; P=0.045$ ), whereas the E-fed rats (absolute mean difference $13 \%$; $95 \%$ CI: $6 \%-19 \% ; P=0.071$ ) tended to reduce liver weight compared with the $\mathrm{C}$ group (Table 1).
Serum, Hepatic, and Fecal Concentrations of Triglyceride and Cholesterol. Compared with the $\mathrm{C}$ group, feeding the $\mathrm{F}$ diet reduced serum triglyceride levels 73\% (95\% CI: $67 \%-79 \% ; P<0.001$ ), 39\% when ezetimibe

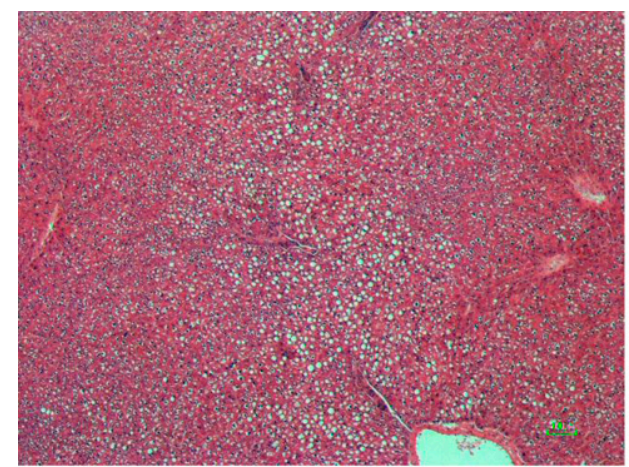

A

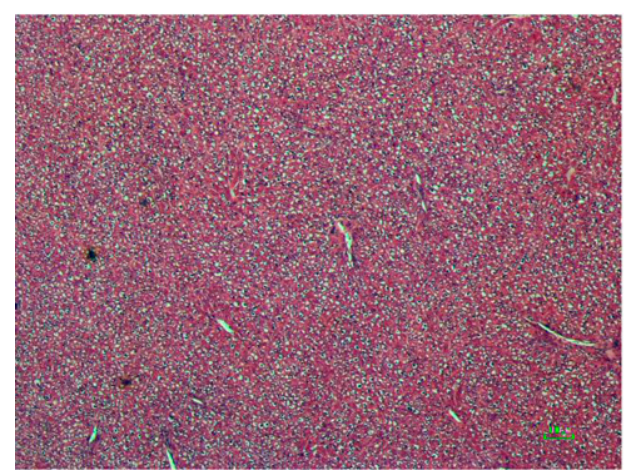

C

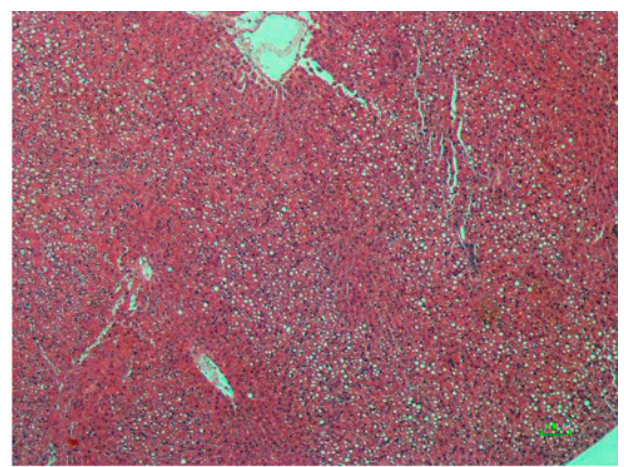

B

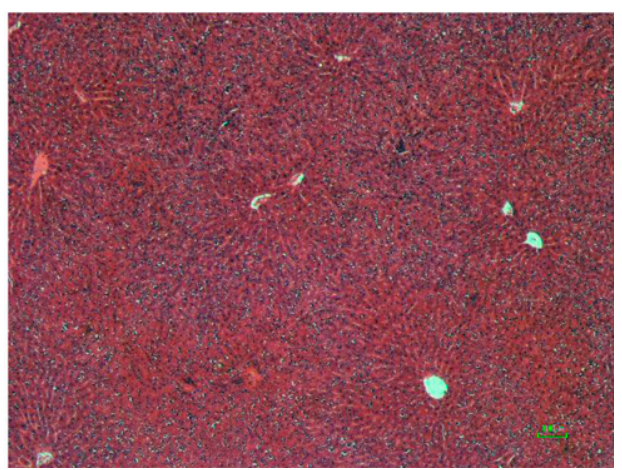

D
Fig. 1. Histologic examination in rats after 4 weeks of each diet. Liver H\&E staining is presented as follows: $\mathrm{C}$-fed (A), F-fed (B), E-fed (C), and F+E-fed (D) rats. Representative images are shown. Scale bar, $100 \mu \mathrm{m}$. 

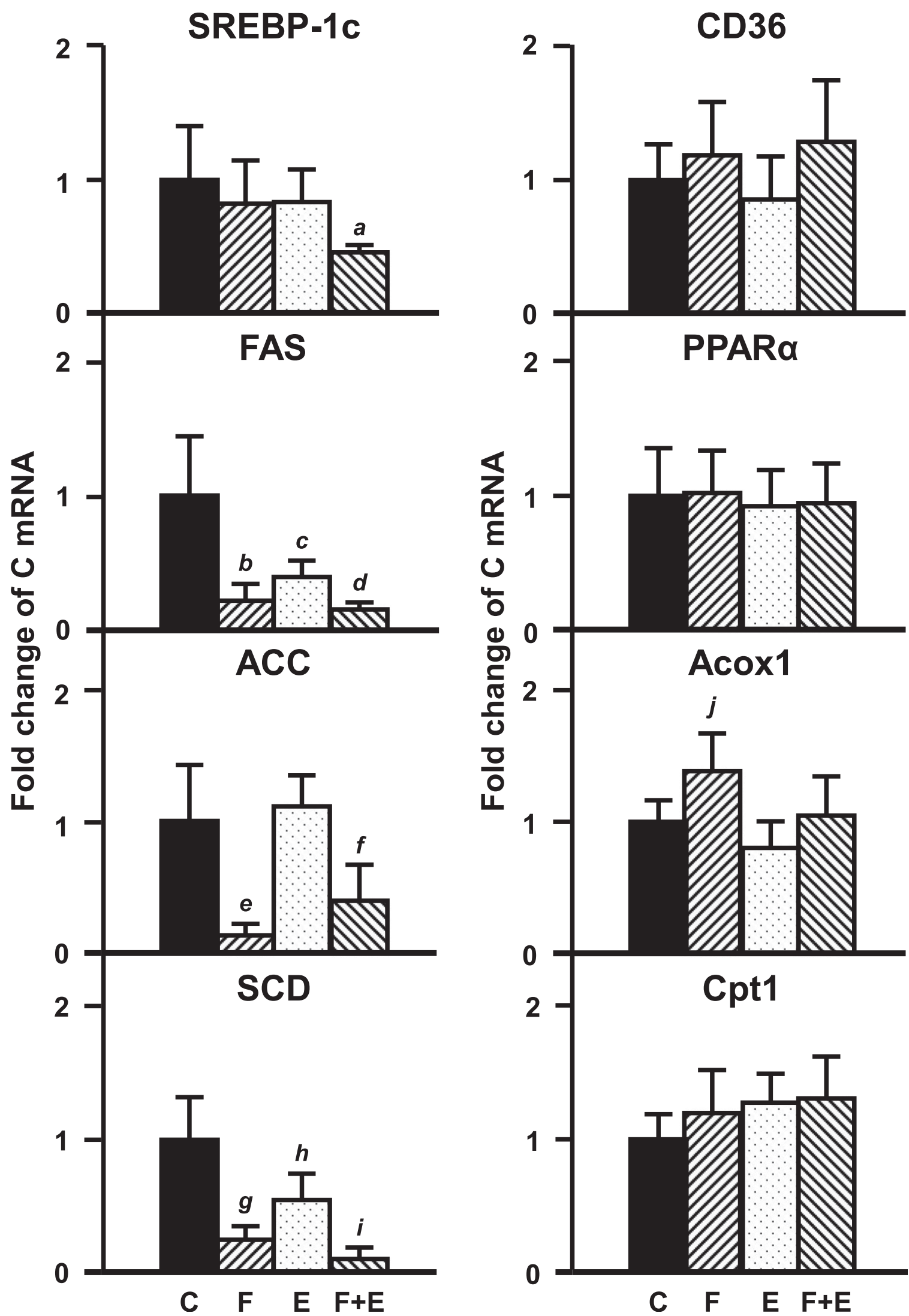

Fig. 2. mRNA expression of fatty acid-related genes in rat liver after 4 weeks of each diet. Total hepatic RNA was isolated from rats, and mRNA levels were quantified by real-time PCR as described in Materials and Methods. Data are presented as means \pm S.D. (each group, $n=7$ animals). Rats were allocated to four different diets containing 1) $10 \%$ soybean oil (C; control group), 2) $10 \%$ menhaden oil (F; fish oil group), 3) $10 \%$ soybean oil plus $0.005 \%$ ezetimibe (E; ezetimibe group), and 4$) 10 \%$ fish oil plus $0.005 \%$ ezetimibe ( $\mathrm{F}+\mathrm{E}$; fish oil plus ezetimibe group) for 4 weeks. Data were analyzed by one-way ANOVA, followed by Tukey's post hoc test. Significance was set at $P<0.05 . a, P=0.005$, significant difference from C; $b, P<0.001$, significant 
was added to the $\mathrm{C} \operatorname{diet}(95 \% \mathrm{CI}: 19 \%-58 \% ; P=0.033)$, and $83 \%$ when ezetimibe was added to the $\mathrm{F}$ diet (95\% CI: $78 \%-88 \% ; P<0.001)$. Serum total cholesterol and the HDL cholesterol were approximately the same in the rats fed the $\mathrm{C}$ diet and those that had ezetimibe added to the $\mathrm{C}$ diet, but the $\mathrm{F}$ diet reduced total cholesterol $33 \%$ (95\% CI: $24 \%-41 \% ; P<$ $0.001)$ and HDL cholesterol $41 \%$ (95\% CI: $34 \%-48 \%$; $P<$ 0.001 ), and when ezetimibe was added to the $\mathrm{F}$ diet, total cholesterol was reduced $36 \%$ (95\% CI: $27 \%-46 \% ; P<0.001$ ) and HDL cholesterol was reduced $23 \%$ (95\% CI: $13 \%-32 \% ; P=$ 0.002). Serum HDL cholesterol was $45 \%$ (95\% CI: $38 \%-51 \%$; $P<0.001)$ lower in rats fed the $\mathrm{F}$ diet than the $\mathrm{E}$ diet. The content of triglyceride in the whole liver was decreased 48\% (95\% CI: $31 \%-66 \% ; P=0.026$ ) when the $\mathrm{C}$ diet was replaced with the $\mathrm{F}$ diet, but liver triglyceride concentrations were not decreased. Ezetimibe did not decrease the concentrations and total content of triglyceride in the liver of the rats fed the $\mathrm{C}$ diet, but ezetimibe markedly decreased liver triglyceride concentrations $84 \%$ (95\% CI: 80\%-88\%; $P<0.001$ ) and whole-liver triglyceride content $86 \%$ (95\% CI: $82 \%-91 \%$; $P<0.001$ ) when added to the $\mathrm{F}$ diet. Similarly, liver cholesterol concentrations and whole-liver cholesterol content were not decreased when the $\mathrm{C}$ diet was replaced with the $\mathrm{F}$ diet or when ezetimibe was added to the $\mathrm{C}$ diet, but ezetimibe markedly decreased liver cholesterol concentrations 86\% (95\% CI: $83 \%-89 \% ; P=0.001)$ and whole-liver cholesterol content $88 \%(95 \% \mathrm{CI}: 85 \%-91 \% ; P<0.001)$ when added to the diet of the F-fed rats. Fecal triglycerides were reduced 47\% (95\% CI: $33 \%-61 \% ; P=0.002$ ) when the $\mathrm{C}$ diet was replaced with the $\mathrm{F}$ diet, and ezetimibe did not reduce the triglycerides in the feces of the rats fed the $\mathrm{C}$ diet, but ezetimibe reduced fecal triglyceride levels $55 \%$ (95\% CI: $44 \%-66 \% ; P<0.001)$ when added to the $\mathrm{F}$ diet. Fecal excretion of cholesterol was $41 \%$ (95\% CI: $25 \%-57 \% ; P=0.005$ ) higher in rats fed the F-diet and tended to be higher (absolute mean difference $28 \%$; 95\% CI: $9 \%-48 \% ; P=0.078$ ) in rats fed the $\mathrm{E}$ diet than the $\mathrm{C}$ diet; however, this increase in fecal cholesterol levels in the $\mathrm{F}$ group was attenuated in the $\mathrm{F}+\mathrm{E}$ group (Table 1).

Histopathology. To determine differences in hepatic steatosis histologically, H\&E was conducted. Photomicrographs of H\&E-stained liver sections from the C-fed and E-fed rats (Figs. 1A and 1C) demonstrated predominant macrovesicular steatosis in the hepatic lobules, and the degree of steatosis was mild or moderate. H\&E-stained liver section from the F-diet rat (Fig. 1B) showed less fat deposition compared with the C-fed and E-fed rats. Furthermore, the F+E-fed rat (Fig. 1D) hardly showed fat deposition in the liver. These results were similar to whole-liver triglyceride content.

Hepatic Expression of Fatty Acid-Related Genes. The mRNA expression of SREBP-1c and its target lipogenic enzymes was quantified. The mRNA expression of SREBP-1c was decreased $55 \%(95 \% \mathrm{CI}: 51 \%-59 \% ; P=0.005)$ in the $\mathrm{F}+\mathrm{E}$ diet. The mRNA expression of FAS, ACC, and SCD was decreased $78 \%$ (95\% CI: 70\%-86\%; $P<0.001$ ), $87 \%$ (95\% CI: $81 \%-92 \% ; P<0.001)$, and $76 \%$ (95\% CI: $69 \%-83 \% ; P<0.001)$ in the F-fed rats and 85\% (95\% CI: 79\%-90\%; $P<0.001)$,
60\% (95\% CI: 40\%-80\%; $P=0.003$ ), and $90 \%(95 \% \mathrm{CI}$ : $83 \%-97 \% ; P<0.001)$ in the $\mathrm{F}+\mathrm{E}$ diet. However, there were no differences in the mRNA expression of these genes between the $\mathrm{F}$ group and the $\mathrm{F}+\mathrm{E}$ group. The mRNA expression of the fatty acid oxidation genes was determined. The mRNA expression of $\operatorname{PPAR} \alpha$ target gene Acox1 was increased $38 \%$ (95\% CI: $18 \%-59 \% ; P=0.038)$ in the $\mathrm{F}$-fed rats compared with the C-fed rats (Fig. 2).

Hepatic Expression of Cholesterol Homeostatic Genes and Bile Acid Synthetic Enzymes. Cholesterol levels are maintained by the balance of uptake, synthesis, catabolism, and export. Compared with the C-fed rats, mRNA expression of cholesterol efflux transporters Abcg5 and Abcg8 was induced 1380\% (95\% CI: 965\%-1795\%; $P<0.001)$ and $442 \%$ (95\% CI: $270 \%-613 \% ; P<0.001)$ in the F+E diet. The mRNA expression of phospholipid transporter Mdr2 was increased $92 \%(95 \% \mathrm{CI}: 45 \%-140 \% ; P=0.006)$ in the $\mathrm{F}+\mathrm{E}-$ fed rats compared with the $\mathrm{C}$-fed rats. The mRNA expression of cholesterol synthesis gene HMG-CoA synthase was increased $481 \%$ (95\% CI: $391 \%-571 \% ; P<0.001)$ in the $\mathrm{F}+\mathrm{E}-$ fed rats compared with the C-fed rats. The mRNA expression of Cyp7a1, the rate-limiting enzyme of bile acid synthesis, was decreased $66 \%$ (95\% CI: $51 \%-82 \% ; P=0.022)$ in the F+E-fed rats compared with the F-fed rats. The mRNA expression of Cyp27a1, a key enzyme in the alternative acidic pathway of bile acid synthesis, tended to be decreased (absolute mean difference 19\%; 95\% CI: $10 \%-27 \% ; P=0.065)$ in the $\mathrm{F}+\mathrm{E}$-fed rats compared with the $\mathrm{F}$-fed rats. In contrast, compared with the $\mathrm{C}$-fed and the F-fed rats, the mRNA expression of the cholic acid-synthesizing enzyme Cyp8b1 was increased 89\% (95\% CI: $61 \%-117 \% ; P<0.001)$ and $45 \%$ (95\% CI: $23 \%-67 \% ; P=0.006$ ) in the $\mathrm{F}+\mathrm{E}-\mathrm{fed}$ rats, respectively (Fig. 3).

Jejunal Expression of Cholesterol and Bile Acid Metabolism-Related Genes. The mRNA expression of Abcg5 and Abcg8 in jejunum was increased $244 \%(95 \% \mathrm{CI}$ : $77 \%-411 \% ; P=0.006)$ and $841 \%(95 \% \mathrm{CI}: 302 \%-1381 \% ; P=$ 0.008 ) in the $\mathrm{F}+\mathrm{E}$-fed rats compared with the $\mathrm{C}$-fed rats. The mRNA expression of bile acid uptake transporter Asbt was increased $970 \%$ (95\% CI: $345 \%-1602 \%$; $P=0.004$ ) in the F+Efed rats compared with the C-fed rats (Table 2). The mRNA expression of the HDL receptor SR-B1 was decreased $56 \%$ (95\% CI: $48 \%-65 \% ; P=0.044)$ in the F-fed rats compared with the E-fed rats.

\section{Discussion}

To date, studies have demonstrated that weight loss through dietary regulation and exercise is effective for the treatment of NAFLD. However, the therapeutic efficacy of drugs against NAFLD has not been verified (Huang et al., 2005; Vilar-Gomez et al., 2015). Therefore, to investigate the combined effects of dietary fish oil and ezetimibe on lipid metabolism in rats, four different diets were fed to rats for 4 weeks. On the basis of the data in previous studies, a diet containing $10 \%$ soybean oil was used to induce fatty liver in the control group (Ataide et al., 2009; Farias Santos et al.,

difference from $\mathrm{C} ; c, P=0.001$, significant difference from $\mathrm{C} ; d, P<0.001$, significant difference from $\mathrm{C} ; e, P<0.001$, significant difference from $\mathrm{C}$; $f$, $P=0.003$, significant difference from $\mathrm{C} ; g, P<0.001$, significant difference from $\mathrm{C} ; h, P=0.001$, significant difference from $\mathrm{C} ; i, P<0.001$, significant difference from $\mathrm{C} ; j, P=0.038$, significant difference from $\mathrm{C}$. 

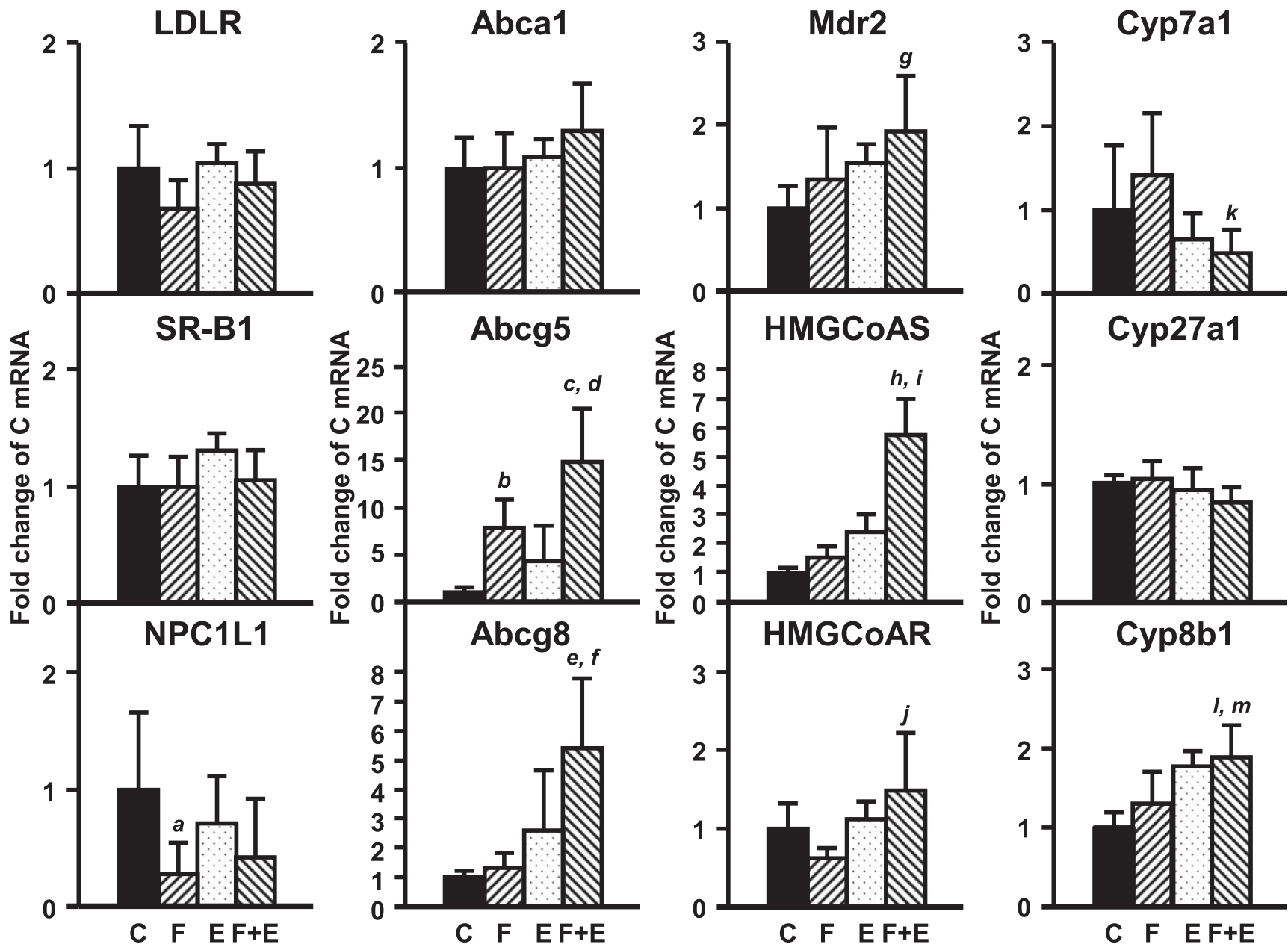

Fig. 3. mRNA expression of cholesterol homeostatic genes, bile acid synthetic enzymes, and phospholipid transporter in rat liver after 4 weeks of each diet. Total hepatic RNA was isolated from rats, and mRNA levels were quantified by real-time PCR as described in Materials and Methods. Data are presented as means \pm S.D. (each group, $n=7$ animals). Rats were allocated to four different diets containing 1) 10\% soybean oil (C; control group), 2) $10 \%$ menhaden oil (F; fish oil group), 3) $10 \%$ soybean oil plus $0.005 \%$ ezetimibe (E; ezetimibe group), and 4) $10 \%$ fish oil plus $0.005 \%$ ezetimibe (F+E; fish oil plus ezetimibe group) for 4 weeks. Data were analyzed by one-way ANOVA, followed by Tukey's post hoc test. Significance was set at $P<0.05$. $a, P=$ 0.049 , significant difference from $\mathrm{C} ; b, P=0.01$, significant difference from $\mathrm{C} ; c, P<0.001$, significant difference from $\mathrm{C} ; d, P=0.008$, significant difference from $\mathrm{F} ; e, P<0.001$, significant difference from $\mathrm{C} ; f, P<0.001$, significant difference from $\mathrm{F} ; g, P=0.006$, significant difference from $\mathrm{C} ; h, P<$ 0.001 , significant difference from $\mathrm{C} ; i, P<0.001$, significant difference from $\mathrm{F} ; j, P=0.004$, significant difference from $\mathrm{F} ; k, P=0.022$, significant difference from $\mathrm{F} ; l, P<0.001$, significant difference from $\mathrm{C} ; m, P=0.006$, significant difference from $\mathrm{F}$. LDLR, low-density lipoprotein receptor.

2015). The present study revealed that the combined administration of ezetimibe, a medication used for treating dyslipidemia, and fish oil, which is rich in n-3 polyunsaturated fatty acids, significantly reduced the hepatic levels of lipids and suppressed the formation of lipid droplets in rats, as revealed by histologic analysis. This suppression was stronger in the

TABLE 2

Gene expression of cholesterol and bile acid metabolism-related genes in jejunum.

Genes were categorized according to biologic mechanism. Data are presented as means \pm S.D. (each group, $n=7$ animals). Data were analyzed by one-way ANOVA, followed by Tukey's post hoc test. Significance was set at $P<0.05$. The 95\% CI and the $P$-value are listed.

\begin{tabular}{|c|c|c|c|c|c|c|c|}
\hline \multirow[b]{2}{*}{ Category } & \multirow[b]{2}{*}{ Gene Name } & \multicolumn{4}{|c|}{ Fold Change of $\mathrm{C}$} & \multirow[b]{2}{*}{$95 \% \mathrm{CI}$} & \multirow[b]{2}{*}{$P$-Value } \\
\hline & & $\mathrm{C}$ & $\mathrm{F}$ & $\mathrm{E}$ & $\mathrm{F}+\mathrm{E}$ & & \\
\hline \multicolumn{8}{|c|}{ Cholesterol homeostasis } \\
\hline & NPC1L1 & $1.0 \pm 0.4$ & $1.1 \pm 0.4$ & $1.8 \pm 1.1$ & $1.2 \pm 0.7$ & $-39 \%$ to $70 \%$ & $0.978, \mathrm{C}$ vs. $\mathrm{F}+\mathrm{E}$ \\
\hline & Abca 1 & $1.0 \pm 0.9$ & $0.9 \pm 0.6$ & $0.6 \pm 0.2$ & $0.8 \pm 0.2$ & $8 \%-36 \%$ & $0.891, \mathrm{C}$ vs. $\mathrm{F}+\mathrm{E}$ \\
\hline & Abcg5 & $1.0 \pm 0.4$ & $2.1 \pm 0.8$ & $1.9 \pm 0.5$ & $3.4 \pm 2.3$ & $77 \%-411 \%$ & $0.006, \mathrm{C}$ vs. $\mathrm{F}+\mathrm{E}$ \\
\hline & Abcg8 & $1.0 \pm 0.4$ & $6.4 \pm 3.3$ & $3.8 \pm 3.7$ & $9.4 \pm 7.3$ & $302 \%-1381 \%$ & $0.008, \mathrm{C}$ vs. $\mathrm{F}+\mathrm{E}$ \\
\hline & LDLR & $1.0 \pm 0.4$ & $1.1 \pm 0.4$ & $1.4 \pm 0.6$ & $1.0 \pm 0.3$ & $-16 \%$ to $27 \%$ & $0.996, \mathrm{C}$ vs. $\mathrm{F}+\mathrm{E}$ \\
\hline & SR-B1 & $1.0 \pm 0.4$ & $0.6 \pm 0.2$ & $1.5 \pm 0.9$ & $0.7 \pm 0.5$ & $48 \%-65 \%$ & $0.044, \mathrm{~F}$ vs. $\mathrm{E}$ \\
\hline \multicolumn{8}{|c|}{ Bile acid transporter } \\
\hline & Asbt & $1.0 \pm 0.5$ & $7.1 \pm 3.5$ & $4.1 \pm 2.3$ & $10.7 \pm 8.5$ & $345 \%-1602 \%$ & $0.004, \mathrm{C}$ vs. $\mathrm{F}+\mathrm{E}$ \\
\hline & Ost $\alpha$ & $1.0 \pm 0.5$ & $1.2 \pm 0.6$ & $1.9 \pm 1.0$ & $1.6 \pm 1.3$ & $-36 \%$ to $154 \%$ & $0.612, \mathrm{C}$ vs. $\mathrm{F}+\mathrm{E}$ \\
\hline & Ost $\beta$ & $1.0 \pm 0.3$ & $1.4 \pm 0.8$ & $2.3 \pm 1.1$ & $1.7 \pm 1.1$ & $-9 \%$ to $156 \%$ & $0.419, \mathrm{C}$ vs. $\mathrm{F}+\mathrm{E}$ \\
\hline
\end{tabular}

LDLR, low-density lipoprotein receptor. 
$\mathrm{F}+\mathrm{E}$ group than the $\mathrm{F}$ group and the group that received only ezetimibe (Fig. 1; Table 1).

Dyslipidemia is observed in some patients with NAFLD. Ezetimibe, a lipid-lowering agent, is considered to be a therapeutic option for NAFLD because it inhibits NPC1L1mediated cholesterol uptake in the small intestine (Yamagishi et al., 2006; Ostovaneh et al., 2015). NAFLD improves when the production of chylomicrons is reduced, and because cholesterol is necessary for chylomicron synthesis in the small intestine, the inhibition of cholesterol uptake by ezetimibe is thought to be the underlying mechanism for the improvement in NAFLD after ezetimibe treatment (de Bari et al., 2012). A study demonstrated that the improvement in the serum and hepatic levels of triglycerides and cholesterol and fatty liver histology was more significant in Zucker rats fed on a high-fat diet supplemented with ezetimibe in comparison with that of the rats that were fed on a high-fat diet only (Deushi et al., 2007). However, the levels of serum and hepatic cholesterol did not show any improvement in the group that received only ezetimibe in this study, and this was also true for the fatty liver. However, the serum levels of triglycerides did show improvements (Fig. 1; Table 1). The difference in the composition of fatty acids in the feed and the dosage of ezetimibe may have resulted in the discrepancy between the results of the study by Deushi and coworkers and those obtained in the present study.

It is considered that n-3 polyunsaturated fatty acid (PUFA), which is abundant in fish oil, can effectively reduce the serum levels of triglycerides and prevent cardiovascular disorders (Harris et al., 1988; Yagi et al., 2017). A study reported that the diets of patients with NAFLD contain large amounts of saturated fatty acids and cholesterol and small amounts of $n-3$ PUFA (Musso et al., 2003). Another study reported a decrease in the levels of $n-3$ PUFA and an increase in the ratio of $n-6$ fatty acids to n-3 fatty acids in the hepatic triglycerides of patients with NASH (Puri et al., 2007). Although fish oil ameliorates obesity, insulin resistance, and fatty liver in rodents, no consensus has been reached regarding its effect on the levels of hepatic enzymes and fatty liver in human subjects (Sekiya et al., 2003; Lombardo et al., 2007; Tanaka et al., 2008; Sanyal et al., 2014). In this study, the serum levels of triglycerides, cholesterol, and HDL cholesterol were reduced in the F group. However, the difference in the hepatic triglyceride levels between the $\mathrm{F}$ group and the control group was not statistically significant, and the levels of hepatic triglycerides were lower in the $\mathrm{F}$ group than in the group that received only ezetimibe (Table 1). Considering the decrease in serum HDL cholesterol in the F group, hepatic and jejunal mRNA expression of Abca1, which is involved in HDL particle formation, was not changed among all groups. Compared with the E group, the mRNA expression of the HDL receptor SR-B1 was not induced in the liver but was reduced in the jejunum in the F group, which likely contributed to the decreased serum HDL cholesterol levels by inhibiting its absorption (Bietrix et al., 2006; Duong et al., 2006) (Fig. 3; Table 2).

The hepatic levels of triglycerides and cholesterol were not reduced in the $\mathrm{F}$ group and the group that received only ezetimibe. However, for the $\mathrm{F}+\mathrm{E}$ group, the hepatic levels of lipids were markedly reduced, and the fatty liver histology also showed improvements. The above findings suggested that fish oil and ezetimibe worked synergistically when administered together (Fig. 1; Table 1). The expression of the genes related to lipid metabolism were quantified in this study for elucidating the mechanism underlying the marked suppression in the hepatic lipid levels by fish oil and ezetimibe. The results obtained in this study corresponded to those of existing studies. It was observed that the expression of the fatty acid synthesis genes FAS, ACC, and SCD in the liver decreased in the F group; however, no further reduction was observed for the F+E group (Katsurada et al., 1990; Ntambi, 1992; Xu et al., 1999). Analysis of the expression of the genes associated with $\beta$-oxidation revealed that only the expression of Acox1 was increased in the F group (Fig. 2).

The notable changes in gene expression observed in this study were the increased expression of the cholesterol excretion transporters Abcg5/g8 in the liver and the small intestine and the increased hepatic expression of $\mathrm{Mdr} 2$ in the $\mathrm{F}+\mathrm{E}$ group (Fig. 3; Table 2). We have previously reported that the mRNA expression of Abcg5/g8 is increased in the liver and small intestine of mice fed on fish oil (Kamisako et al., 2012). Another study reported that the expression of Abcg5/g8 is increased in the liver of mice that were administered ezetimibe (Altemus et al., 2014). These findings suggest that the combined administration of fish oil and ezetimibe has an additive effect in increasing the expression of Abcg5/g8. It has been observed that a high-fat, high-cholesterol diet causes fatty liver and induces progression to steatohepatitis in rodents (Côté et al., 2013; Savard et al., 2013). In a recent study, a high-fat, high-cholesterol diet was fed to wild-type mice and transgenic mice with liver-specific overexpression of NPC1L1 over the course of 2 weeks (Toyoda et al., 2019). The study reported a marked formation of fatty liver in the transgenic mice but not in their wild-type counterparts. It was additionally observed that Mdr2 is necessary for the Abcg5/g8-mediated secretion of hepatic cholesterol into the bile (Langheim et al., 2005). These findings together with the fact that there was no significant difference in the hepatic expression of fatty acid synthesis genes between the $\mathrm{F}$ group and the $\mathrm{F}+\mathrm{E}$ group suggest that the increased hepatic expression of Abcg5/g8 and Mdr2 in the $\mathrm{F}+\mathrm{E}$ group contributed to the marked improvement in the fatty liver (Figs. 1 and 3).

A recent study reported the phenomenon of transintestinal cholesterol excretion, in which cholesterol is directly excreted from the epithelial cells of the small intestine into the intestine (van der Velde et al., 2008). The transintestinal cholesterol excretion pathway is different from that of biliary cholesterol excretion. In this study, the increase in the levels of fecal cholesterol was higher in the $\mathrm{F}$ group than in the control group. On the other hand, there was no increase in the fecal cholesterol levels of the $\mathrm{F}+\mathrm{E}$ group. It was therefore considered that the increased expression of Abcg5/g8 in the liver instead of in the small intestine contributed to the reduction in the levels of serum and hepatic lipids and the improvement in fatty liver. Cyp8b1 is required for synthesis of cholic acid, which plays a role in intestinal cholesterol absorption. Asbt is responsible for the absorption of bile acids from the small intestine. The expression of these two genes was increased in the $\mathrm{F}+\mathrm{E}$ group. This, together with cholesterol reabsorption, may explain the observation that the fecal cholesterol levels were not increased in the $\mathrm{F}+\mathrm{E}$ group (Fig. 3; Tables 1 and 2) (Murphy et al., 2005; van de Peppel et al., 2019).

There may be some possible limitations in this study. For gene expression analysis, we only quantified mRNA levels of 
fatty acid, cholesterol, and bile acid metabolism-related genes. mRNA levels do not always directly correlate with protein levels because of translational and post-translational regulation. Therefore, Western blot analysis should be performed to determine whether changes in mRNA expression corresponded with protein levels in future research.

In conclusion, the combined administration of fish oil and ezetimibe significantly decreased the serum and hepatic levels of triglycerides and cholesterol and markedly improved fatty liver histology. It was considered that the marked improvement in fatty liver histology in the $\mathrm{F}+\mathrm{E}$ group was associated with the increase in the expression of Abcg5/g8 and Mdr2 in the liver. The results of this study suggest that the combination therapy of ezetimibe and fish oil agents may be effective in treating NAFLD.

\section{Acknowledgments}

The authors would like to thank Yasumitsu Akahoshi for technical assistance.

\section{Authorship Contributions}

Participated in research design: Tanaka, Kamisako.

Conducted experiments: Tanaka, Ikeda.

Contributed new reagents or analytic tools: Tanaka, Ikeda, Ogawa,

Kamisako.

Performed data analysis: Tanaka.

Wrote or contributed to the writing of the manuscript: Tanaka, Kamisako.

\section{References}

Altemus JB, Patel SB, and Sehayek E (2014) Liver-specific induction of Abcg5 and Abcg8 stimulates reverse cholesterol transport in response to ezetimibe treatment. Metabolism 63:1334-1341.

Anushiravani A, Haddadi N, Pourfarmanbar M, and Mohammadkarimi V (2019) Treatment options for nonalcoholic fatty liver disease: a double-blinded randomized placebo-controlled trial. Eur J Gastroenterol Hepatol 31:613-617.

Aso Y, Kato K, Sakurai S, Kishi H, Shimizu M, Jojima T, Iijima T, Maejima Y, Shimomura K, and Usui I (2019) Impact of dapagliflozin, an SGLT2 inhibitor, on serum levels of soluble dipeptidyl peptidase- 4 in patients with type 2 diabetes and non-alcoholic fatty liver disease. Int $J$ Clin Pract 73:e13335.

Armstrong MJ, Gaunt P, Aithal GP, Barton D, Hull D, Parker R, Hazlehurst JM, Guo K, Abouda G, Aldersley MA, et al.; LEAN trial team (2016) Liraglutide safety and efficacy in patients with non-alcoholic steatohepatitis (LEAN): a multicentre, double-blind, randomised, placebo-controlled phase 2 study. Lancet 387:679-690.

Ataide TR, Oliveira SL, Silva FM, Vitorino Filha LGC, Tavares MCN, and Sant'Ana AEG (2009) Toxicological analyses of the chronic consumption of diheptanoin and triheptanoin in rats. Int J Food Sci Technol 44:484-492.

Ballatori N, Christian WV, Lee JY, Dawson PA, Soroka CJ, Boyer JL, Madejczyk MS, and Li N (2005) OSTalpha-OSTbeta: a major basolateral bile acid and steroid transporter in human intestinal, renal, and biliary epithelia. Hepatology $\mathbf{4 2}$ 1270-1279.

Baumgardner JN, Shankar K, Hennings L, Badger TM, and Ronis MJ (2008) A new model for nonalcoholic steatohepatitis in the rat utilizing total enteral nutrition to overfeed a high-polyunsaturated fat diet. Am J Physiol Gastrointest Liver Physiol 294:G27-G38.

Bietrix F, Yan D, Nauze M, Rolland C, Bertrand-Michel J, Coméra C, Schaak S, Barbaras R, Groen AK, Perret B, et al. (2006) Accelerated lipid absorption in mice overexpressing intestinal SR-BI. J Biol Chem 281:7214-7219.

Boone LR, Brooks PA, Niesen MI, and Ness GC (2011) Mechanism of resistance to dietary cholesterol. J Lipids 2011:101242.

Chalasani N, Younossi Z, Lavine JE, Charlton M, Cusi K, Rinella M, Harrison SA, Brunt EM, and Sanyal AJ (2018) The diagnosis and management of nonalcoholic fatty liver disease: practice guidance from the American Association for the Study of Liver Diseases. Hepatology 67:328-357.

Chen Q, Gruber H, Pakenham C, Ratnayake WM, and Scoggan KA (2009) Dietary phytosterols and phytostanols alter the expression of sterol-regulatory genes in SHRSP and WKY inbred rats. Ann Nutr Metab 55:341-350.

Côté I, Ngo Sock ET, Lévy É, and Lavoie JM (2013) An atherogenic diet decreases liver FXR gene expression and causes severe hepatic steatosis and hepatic cholesterol accumulation: effect of endurance training. Eur J Nutr 52:1523-1532.

de Bari O, Neuschwander-Tetri BA, Liu M, Portincasa P, and Wang DQ (2012) Ezetimibe: its novel effects on the prevention and the treatment of cholesterol gallstones and nonalcoholic Fatty liver disease. J Lipids 2012:302847.

Deushi M, Nomura M, Kawakami A, Haraguchi M, Ito M, Okazaki M, Ishii H, and Yoshida M (2007) Ezetimibe improves liver steatosis and insulin resistance in obese rat model of metabolic syndrome. FEBS Lett 581:5664-5670.

Duong PT, Collins HL, Nickel M, Lund-Katz S, Rothblat GH, and Phillips MC (2006) Characterization of nascent HDL particles and microparticles formed by ABCA1mediated efflux of cellular lipids to apoA-I. J Lipid Res 47:832-843.
Fakhrzadeh H, Ghaderpanahi M, Sharifi F, Mirarefin M, Badamchizade Z, Kamrani AA, and Larijani B (2010) The effects of low dose n-3 fatty acids on serum lipid profiles and insulin resistance of the elderly: a randomized controlled clinical trial. Int $J$ Vitam Nutr Res 80:107-116.

Farias Santos J, Suruagy Amaral M, Lima Oliveira S, Porto Barbosa J, Rego Cabral C Jr., Sofia Melo I, Bezerra Bueno N, Duarte Freitas J, Goulart Sant'ana A, and Rocha Ataíde T (2015) Dietary intake of ain-93 standard diet induces Fatty liver with altered hepatic fatty acid profile in Wistar rats. Nutr Hosp 31: 2140-2146

Folch J, Lees M, and Sloane Stanley GH (1957) A simple method for the isolation and purification of total lipides from animal tissues. J Biol Chem 226:497-509.

Gao X, Zeng Y, Liu S, and Wang S (2013) Acute stress show great influences on liver function and the expression of hepatic genes associated with lipid metabolism in rats. Lipids Health Dis 12:118.

Harris WS, Connor WE, Alam N, and Illingworth DR (1988) Reduction of postprandial triglyceridemia in humans by dietary n-3 fatty acids. J Lipid Res 29 : 1451-1460.

Huang MA, Greenson JK, Chao C, Anderson L, Peterman D, Jacobson J, Emick D, Lok AS, and Conjeevaram HS (2005) One-year intense nutritional counseling results in histological improvement in patients with non-alcoholic steatohepatitis: a pilot study. Am J Gastroenterol 100:1072-1081.

Kamisako T, Tanaka Y, Ikeda T, Yamamoto K, and Ogawa H (2012) Dietary fish oil regulates gene expression of cholesterol and bile acid transporters in mice. Hepatol Res 42:321-326.

Katsurada A, Iritani N, Fukuda H, Matsumura Y, Nishimoto N, Noguchi T, and Tanaka $\mathrm{T}$ (1990) Effects of nutrients and hormones on transcriptional and post-transcriptional regulation of acetyl-CoA carboxylase in rat liver. Eur J Biochem 190:435-441.

Kawashima Y, Eguchi Y, Yamazaki T, Karahashi M, Kawai H, and Kudo N (2018) Reduction in secretion of very low density lipoprotein-triacylglycerol by a matrix metalloproteinase inhibitor in a rat model of diet-induced hypertriglyceridemia. $J$ Pharmacol Exp Ther 366:194-204.

Langheim S, Yu L, von Bergmann K, Lütjohann D, Xu F, Hobbs HH, and Cohen JC (2005) ABCG5 and ABCG8 require MDR2 for secretion of cholesterol into bile. $J$ Lipid Res 46:1732-1738.

Lombardo YB, Hein G, and Chicco A (2007) Metabolic syndrome: effects of n-3 PUFAs on a model of dyslipidemia, insulin resistance and adiposity. Lipids 42:427-437.

Loomba R, Sirlin CB, Ang B, Bettencourt R, Jain R, Salotti J, Soaft L, Hooker J, Kono Y, Bhatt A, et al.; San Diego Integrated NAFLD Research Consortium (SINC) (2015) Ezetimibe for the treatment of nonalcoholic steatohepatitis: assessment by novel magnetic resonance imaging and magnetic resonance elastography in a randomized trial (MOZART trial). Hepatology 61:1239-1250.

Madsen A, Bozickovic O, Bjune JI, Mellgren G, and Sagen JV (2015) Metformin inhibits hepatocellular glucose, lipid and cholesterol biosynthetic pathways by transcriptionally suppressing steroid receptor coactivator 2 (SRC-2). Sci Rep 5: 16430

Michihara A, Mido M, Matsuoka H, and Mizutani Y (2015) Lower squalene epoxidase and higher scavenger receptor class B type 1 protein levels are involved in reduced serum cholesterol levels in stroke-prone spontaneously hypertensive rats. Biol Pharm Bull 38:1879-1890.

Murphy C, Parini P, Wang J, Björkhem I, Eggertsen G, and Gåfvels M (2005) Cholic acid as key regulator of cholesterol synthesis, intestinal absorption and hepatic storage in mice. Biochim Biophys Acta 1735:167-175.

Musso G, Gambino R, De Michieli F, Cassader M, Rizzetto M, Durazzo M, Fagà E, Silli B, and Pagano G (2003) Dietary habits and their relations to insulin resistance and postprandial lipemia in nonalcoholic steatohepatitis. Hepatology 37:909-916.

Ntambi JM (1992) Dietary regulation of stearoyl-CoA desaturase 1 gene expression in mouse liver. J Biol Chem 267:10925-10930.

Ostovaneh MR, Zamani F, Ansari-Moghaddam A, Sharafkhah M, Saeedian FS, Rohani Z, Khoonsari M, Barzin G, Hemmasi G, Ajdarkosh H, et al. (2015) Nonalcoholic fatty liver: the association with metabolic abnormalities, body mass index and central obesity--a population-based study. Metab Syndr Relat Disord 13: 304-311.

Park H, Shima T, Yamaguchi K, Mitsuyoshi H, Minami M, Yasui K, Itoh Y, Yoshikawa T, Fukui M, Hasegawa G, et al. (2011) Efficacy of long-term ezetimibe therapy in patients with nonalcoholic fatty liver disease. $J$ Gastroenterol 46: 101-107.

Preiss D and Sattar N (2008) Non-alcoholic fatty liver disease: an overview of prevalence, diagnosis, pathogenesis and treatment considerations. Clin Sci (Lond) 115: 141-150.

Puri P, Baillie RA, Wiest MM, Mirshahi F, Choudhury J, Cheung O, Sargeant C, Contos MJ, and Sanyal AJ (2007) A lipidomic analysis of nonalcoholic fatty liver disease. Hepatology 46:1081-1090.

Sanyal AJ, Abdelmalek MF, Suzuki A, Cummings OW, and Chojkier M; EPE-A Study Group (2014) No significant effects of ethyl-eicosapentanoic acid on histologic features of nonalcoholic steatohepatitis in a phase 2 trial. Gastroenterology 147:377-384.e1.

Savard C, Tartaglione EV, Kuver R, Haigh WG, Farrell GC, Subramanian S, Chait A, Yeh MM, Quinn LS, and Ioannou GN (2013) Synergistic interaction of dietary cholesterol and dietary fat in inducing experimental steatohepatitis. Hepatology 57:81-92

Sekiya M, Yahagi N, Matsuzaka T, Najima Y, Nakakuki M, Nagai R, Ishibashi S, Osuga J, Yamada N, and Shimano H (2003) Polyunsaturated fatty acids ameliorate hepatic steatosis in obese mice by SREBP-1 suppression. Hepatology 38: $1529-1539$.

Sun Y, Zhang Y, Li N, Zhang H, Zhou L, and Shao L (2014) Exposure to high levels of glucose increases the expression levels of genes involved in cholesterol biosynthesis in rat islets. Exp Ther Med 8:991-997.

Tanaka N, Sano K, Horiuchi A, Tanaka E, Kiyosawa K, and Aoyama T (2008) Highly purified eicosapentaenoic acid treatment improves nonalcoholic steatohepatitis. $J$ Clin Gastroenterol 42:413-418. 
Toyoda Y, Takada T, Umezawa M, Tomura F, Yamanashi Y, Takeda K, and Suzuki H (2019) Identification of hepatic NPC1L1 as an NAFLD risk factor evidenced by ezetimibe-mediated steatosis prevention and recovery. FASEB Bioadv 1:283-295.

Tu Y, Sun D, Zeng X, Yao N, Huang X, Huang D, and Chen Y (2014) Piperine potentiates the hypocholesterolemic effect of curcumin in rats fed on a high fat diet. Exp Ther Med 8:260-266.

Ushio M, Nishio Y, Sekine O, Nagai Y, Maeno Y, Ugi S, Yoshizaki T, Morino K, Kume S, Kashiwagi A, et al. (2013) Ezetimibe prevents hepatic steatosis induced by a high-fat but not a high-fructose diet. Am J Physiol Endocrinol Metab 305: E293-E304.

van de Peppel IP, Bertolini A, van Dijk TH, Groen AK, Jonker JW, and Verkade HJ (2019) Efficient reabsorption of transintestinally excreted cholesterol is a strong determinant for cholesterol disposal in mice. J Lipid Res 60:1562-1572.

van der Velde AE, Vrins CL, van den Oever K, Seemann I, Oude Elferink RP, van Eck M, Kuipers F, and Groen AK (2008) Regulation of direct transintestinal cholesterol excretion in mice. Am J Physiol Gastrointest Liver Physiol 295: G203-G208.

van Golen RF, Olthof PB, Lionarons DA, Reiniers MJ, Alles LK, Uz Z, de Haan L, Ergin B, de Waart DR, Maas A, et al. (2018) FXR agonist obeticholic acid induces liver growth but exacerbates biliary injury in rats with obstructive cholestasis. $S c i$ Rep 8:16529.

van Heek M, Austin TM, Farley C, Cook JA, Tetzloff GG, and Davis HR (2001) Ezetimibe, a potent cholesterol absorption inhibitor, normalizes combined dyslipidemia in obese hyperinsulinemic hamsters. Diabetes 50:1330-1335.

Vilar-Gomez E, Martinez-Perez Y, Calzadilla-Bertot L, Torres-Gonzalez A, GraOramas B, Gonzalez-Fabian L, Friedman SL, Diago M, and Romero-Gomez M (2015) Weight loss through lifestyle modification significantly reduces features of nonalcoholic steatohepatitis. Gastroenterology 149:367-378, NaN-e15.

Wang J, Gao H, Ke D, Zuo G, Yang Y, Yamahara J, and Li Y (2013) Improvement of liquid fructose-induced adipose tissue insulin resistance by ginger treatment in rats is associated with suppression of adipose macrophage-related proinflammatory cytokines. Evid Based Complement Alternat Med 2013:590376.

Wang X, Sugimoto K, Fujisawa T, Shindo N, Minato S, Kamada Y, Hamano M, Ohishi M, Ikegami H, and Rakugi H (2014) Novel effect of ezetimibe to inhibit the development of non-alcoholic fatty liver disease in Fatty Liver Shionogi mouse. Hepatol Res 44:102-113.

Wei M, Shao Y, Liu QR, Wu QZ, Zhang X, Zhong MW, Liu SZ, Zhang GY, and Hu SY (2018) Bile acid profiles within the enterohepatic circulation in a diabetic rat model after bariatric surgeries. Am J Physiol Gastrointest Liver Physiol 314:G537-G546. Xu J, Nakamura MT, Cho HP, and Clarke SD (1999) Sterol regulatory element binding protein-1 expression is suppressed by dietary polyunsaturated fatty acids. A mechanism for the coordinate suppression of lipogenic genes by polyunsaturated fats. J Biol Chem 274:23577-23583.

Yagi S, Fukuda D, Aihara KI, Akaike M, Shimabukuro M, and Sata M (2017) n-3 polyunsaturated fatty acids: promising nutrients for preventing cardiovascular disease. $J$ Atheroscler Thromb 24:999-1010.

Yamagishi S, Nakamura K, Matsui T, Sato T, and Takeuchi M (2006) Inhibition of intestinal cholesterol absorption by ezetimibe is a novel therapeutic target for fatty liver. Med Hypotheses 66:844-846.

Yuan F, Wang H, Tian Y, Li Q, He L, Li N, and Liu Z (2016) Fish oil alleviated highfat diet-induced non-alcoholic fatty liver disease via regulating hepatic lipids metabolism and metaflammation: a transcriptomic study. Lipids Health Dis 15:20.

Zheng S, Hoos L, Cook J, Tetzloff G, Davis H Jr., van Heek M, and Hwa JJ (2008) Ezetimibe improves high fat and cholesterol diet-induced non-alcoholic fatty liver disease in mice. Eur J Pharmacol 584:118-124.

Address correspondence to: Dr. Yuji Tanaka, Department of Clinical Laboratory Medicine, Kindai University Faculty of Medicine, 377-2, Ohnohigashi, Osakasayama 589-8511, Japan. E-mail: ytanaka@med.kindai.ac.jp 